TRANSACTIONS OF THE

AMERICAN MATHEMATICAL SOCIETY

Volume 353, Number 8, Pages 3193-3207

S 0002-9947(01)02323-6

Article electronically published on April 11, 2001

\title{
IGUSA'S LOCAL ZETA FUNCTIONS OF SEMIQUASIHOMOGENEOUS POLYNOMIALS
}

\author{
W. A. ZÚÑ̃IGA-GALINDO
}

\begin{abstract}
In this paper, we prove the rationality of Igusa's local zeta functions of semiquasihomogeneous polynomials with coefficients in a non-archimedean local field $K$. The proof of this result is based on Igusa's stationary phase formula and some ideas on Néron $\pi$-desingularization.
\end{abstract}

\section{INTRODUCTION}

Let $K$ be a non-archimedean local field, and let $\mathcal{O}_{K}$ be the ring of integers of $K$. Let $\pi$ be a uniformizing parameter of $K$, and let the residue field of $K$ be $\mathbb{F}_{q}$, the finite field with $q=p^{r}$ elements. Let $v$ denote the valuation of $K$ such that $v(\pi)=1$. For $x \in K$, let $|x|_{K}=q^{-v(x)}$. Let $|d x|$ be the Haar measure on $K^{n}$, normalized so that the measure of $\mathcal{O}_{K}^{n}$ is equal to one. Let $f(x) \in K[x]$, $x=\left(x_{1}, . ., x_{n}\right)$. The Igusa local zeta function associated to $f$ is defined by

$$
Z(f, s)=\int_{\mathcal{O}_{K}^{n}}|f(x)|_{K}^{s}|d x|, \quad s \in \mathbb{C}, \operatorname{Re}(s)>0 .
$$

The local zeta function $Z(f, s)$ is a holomorphic function on the semiplane $\operatorname{Re}(s)>$ 0 . In the case of $K$ having characteristic zero, Igusa ([7], [8]) and Denef ([3]) proved that $Z(f, s)$ is a rational function of $q^{-s}$. At the present time, the techniques used by Igusa (resolution of singularities) and Denef (elimination of quantifiers in $\mathbb{Q}_{p}$ ) are not available in positive characteristic, so in this case the rationality of $Z(f, s)$ is still an open problem.

The local zeta function contains information about the number of solutions of the congruence $f(x) \equiv 0 \bmod \pi^{j} \mathcal{O}_{K}$ (see e.g. 4]). More precisely, if

$$
N_{j}:=\operatorname{Card}\left\{x \in\left(\mathcal{O}_{K} / \pi^{j} \mathcal{O}_{K}\right)^{n} \mid f(x) \equiv 0 \bmod \pi^{j} \mathcal{O}_{K}\right\},
$$

and $P(t)$ is the Poincaré series $P(t)=\sum_{j=0}^{\infty} N_{j}\left(q^{-n} t\right)^{j}$, then

$$
Z(f, s)=P\left(q^{-s}\right)-q^{s}\left(P\left(q^{-s}\right)-1\right) .
$$

In this paper, we shall study the local zeta functions of semiquasihomogeneous polynomials with an absolutely algebraically isolated singularity at the origin of $K^{n}$.

Received by the editors June 3, 1997 and, in revised form, May 16, 2000.

2000 Mathematics Subject Classification. Primary 11D79, 11S40, 14G10.

Key words and phrases. Local zeta functions, semiquasihomogeneous polynomials, positive characteristic.

This work was supported by COLCIENCIAS, contract \#063-98. 
Let $f(x)$ be a polynomial with coefficients in $K$, and $V_{f}$ the corresponding $K-$ hypersurface. We call a point $P \in K^{n}$ an absolutely algebraically isolated singularity of $V_{f}(K)$, if the only solution of the system of equations

$$
f(x)=\frac{\partial f}{\partial x_{1}}(x)=\ldots=\frac{\partial f}{\partial x_{n}}(x)=0,
$$

over an algebraic fixed closure of $K$, is the point $P$.

Let $\alpha_{1}, . ., \alpha_{n}$ be $n$ relatively prime and positive integers. A polynomial $f(x) \in$ $K[x]$ is called a quasihomogeneous polynomial of weight $d$ and exponents $\alpha_{1}, . ., \alpha_{n}$, if it satisfies

$$
f\left(t^{\alpha_{1}} x_{1}, . ., t^{\alpha_{n}} x_{n}\right)=t^{d} f(x), \quad \text { for every } t \in K,
$$

and the origin of $K^{n}$ is an absolutely algebraically isolated singularity of the $K$-hypersurface $V_{f}$.

A polynomial $F(x)$ is called a semiquasihomogeneous polynomial if it has the form $f(x)+\sum b_{i} e_{i}(x) \in K[x]$, where $f(x)$ is a quasihomogeneous polynomial, and each monomial $e_{i}(x)=x_{1}^{m_{1}} \ldots x_{n}^{m_{n}}$ satisfies $\sum_{j=1}^{n} \alpha_{j} m_{j}>d$, and the origin of $K^{n}$ is an absolutely algebraically isolated singularity of the $K$-hypersurface $V_{F}$. We call the polynomial $f(x)$ the quasihomogeneous part of $F(x)$.

We put $|\alpha|=\sum_{i} \alpha_{i}$, for any $\alpha=\left(\alpha_{1}, . ., \alpha_{n}\right) \in \mathbb{Z}^{n}$. We use the notation $Z(f, D, s)$ for the integral $\int_{D}|f(x)|_{K}^{s}|d x|$. In the case of $D=\mathcal{O}_{K}^{n}$, we use the simplified notation $Z(f, s)$.

The main result of this paper is the following:

Theorem 3.5. Let $F(x) \in K[x]$ be a semiquasihomogeneous polynomial whose quasihomogeneous part $f(x)$ has weight $d$ and exponents $\alpha_{1}, . ., \alpha_{n}$. Then Igusa's local zeta function of $F(x)$ is a rational function of $q^{-s}$. More precisely,

$$
Z(F, s)=\frac{L\left(q^{-s}\right)}{\left(1-q^{-1} q^{-s}\right)\left(1-q^{-|\alpha|} q^{-d s}\right)},
$$

where $\alpha=\left(\alpha_{1}, \ldots, \alpha_{n}\right)$. Furthermore, the polynomial $L\left(q^{-s}\right)$ can be computed effectively.

If in addition the polynomial $F$ is nondegenerate for its Newton diagram and if $K$ has characteristic zero, then a very different way of calculating $Z(F, s)$ is given in [5. The proof of theorem 3.5 gives an effective method to compute the local zeta functions of semiquasihomogeneous polynomials.

We say that a singular point $\bar{P} \in V_{\bar{f}}\left(\mathbb{F}_{q}\right)$, where $\bar{f}$ is the reduction modulo $\pi$ of $f$, is a non-liftable singularity of the hypersurface $V_{f}$, if for every singular point $Q \in V_{f}(K), Q \in \mathcal{O}_{K}^{n}$, the reduction modulo $\pi$ of $Q$ is different from $\bar{P}$. The proof of theorem 3.5 shows that the numerator of the zeta function $Z(F, s)$ depends on the non-liftable singularites of the closed fiber of the hypersurface $V_{F}$, and the denominator depends on the singularity of the generic fiber of $V_{F}$. More precisely, the denominator depends on Newton's diagram of $F(x)$. In the proof of theorem 3.5 , we use Igusa's formula of stationary phase for $\pi$-adic integrals ([10]) and some ideas on Néron $\pi$-desingularization ([13], sect. 17, 18).

As a consequence of theorem 3.5, we obtain the following three corollaries.

Corollary 3.6. Let $K$ be a global field and $F(x) \in K[x]$ a semiquasihomogeneous polynomial whose quasihomogeneous part $f(x)$ has weight $d$ and exponents $\alpha_{1}, . ., \alpha_{n}$. Then for every non-archimedean valuation $v$ of $K$, Igusa's local zeta function of 
$F(x)$ on the completion $K_{v}$ of $K$ is a rational function of the form (1.1). If $K$ is a number field and $F(x)$ is non-degenerate for its Newton's diagram, then the real parts of the poles of the zeta function $Z(F, s)$ are roots of the Bernstein polynomial of $F(x)$.

For the definition of the Bernstein polynomial and its computation in the nondegenerate case, see reference [2]. The last part of corollary 3.6 is a special case of a more general result due to Loeser (cf. [11], thm. 5.5.1).

Corollary 3.7. Let $K$ be a global field and $\mathcal{O}_{K}$ its ring of integers. Let $F(x) \in$ $\mathcal{O}_{K}[x]$ be a semiquasihomogeneous polynomial whose quasihomogeneous part $f(x)$ has weight $d$ and exponents $\alpha_{1}, \ldots, \alpha_{n}$. Then for every non-archimedean valuation $v$ of $K$, the number of solutions $N_{j}(F, v)$ of the congruence

$$
F(x) \equiv 0 \bmod \pi^{j} \mathcal{O}_{K_{v}},
$$

where $\mathcal{O}_{K_{v}}$ is the ring of integers of the completion $K_{v}$, satisfies

$$
\lim \sup _{j \rightarrow \infty} N_{j}(F, v)^{1 / j} \leq \begin{cases}q^{n-|\alpha| / d} & \text { if }|\alpha| / d \leq 1, \\ q^{n-1} & \text { if }|\alpha| / d>1 .\end{cases}
$$

The zeta functions of plane curves, with only an absolutely analytically irreducible singularity at the origin, have been extensively studied when the characteristic of $K$ is zero, by Igusa ([9]), Meuser ([12]), among others. Let $f(x, y) \in K[x, y]$ be an absolutely analytically irreducible polynomial. Thus there exist $\left(\alpha_{1}, \alpha_{2}\right) \in \mathbb{N}^{2}$, relatively prime integers, and an integer $d$, such that $f(x, y)=f_{d}(x, y)+g(x, y)$ and the monomials $x^{n} y^{m}$ of $f_{d}(x, y)$ and $g(x, y)$ satisfy $\alpha_{1} n+\alpha_{2} m=d$ and $\alpha_{1} n+\alpha_{2} m>d$, respectively. The origin of $K^{2}$ is an absolutely algebraically isolated singularity of the plane curve $V_{f}$. If it is also valid for $V_{f_{d}}$, then $f$ is a semiquasihomogeneous polynomial in the sense of the definition given for us. As a consequence of theorem 3.5, we obtain a precise description of the local zeta function associated with this type of polynomials.

Corollary 3.8. Let $f(x, y) \in K[x, y]$ be an absolutely analytically irreducible polynomial, such that the origin of $K^{2}$ is an absolutely algebraically isolated singularity of the plane curve $V_{f_{d}}$. Then the Igusa local zeta function $Z(f, s)$ is a rational function of $q^{-s}$ of the form (1.1).

\section{Preliminaires}

In 10] Igusa introduced the stationary phase formula for $\pi$-adic integrals and suggested that a closer examination of this formula might lead to a proof of the rationality of $Z(f, s)$ in any characteristic. That suggestion has been our main motivation for this paper. In this section we review Igusa's stationary phase formula and some ideas on Néron $\pi$-desingularization.

We denote by $\bar{x}$ the image of an element of $\mathcal{O}_{K}$ under the canonical homomorphism $\mathcal{O}_{K} \longrightarrow \mathcal{O}_{K} / \pi \mathcal{O}_{K} \cong \mathbb{F}_{q}$, i.e, the reduction modulo $\pi$. Given $f(x) \in \mathcal{O}_{K}[x]$ such that not all its coefficients are in $\pi \mathcal{O}_{K}$, we denote by $\overline{f(x)}$ the polynomial obtained by reducing modulo $\pi$ the coefficients of $f(x)$.

For any commutative $\operatorname{ring} A$ and $f(x) \in A[x]$, we denote by $V_{f}(A)$ the set of $A$-valued points of the hypersurface $V_{f}$ defined by $f$, and by $\operatorname{Sing}_{f}(A)$ the set of 
$A$-valued singular points of $V_{f}$, i.e.,

$$
\operatorname{Sing}_{f}(A)=\left\{x \in A^{n} \mid f(x)=\frac{\partial f}{\partial x_{1}}(x)=\ldots .=\frac{\partial f}{\partial x_{n}}(x)=0\right\} .
$$

We fix a lifting $R$ of $\mathbb{F}_{q}$ in $\mathcal{O}_{K}$. Thus, the set $R$ is mapped bijectively onto $\mathbb{F}_{q}$ by the canonical homomorphism $\mathcal{O}_{K} \longrightarrow \mathcal{O}_{K} / \pi \mathcal{O}_{K}$.

Let $f(x) \in \mathcal{O}_{K}[x]$ be a polynomial in $n$ variables, $P_{1}=\left(y_{1}, \ldots, y_{n}\right) \in \mathcal{O}_{K}^{n}$, and $m_{P_{1}}=\left(m_{1}, \ldots, m_{n}\right) \in \mathbb{N}^{n}$. We call a $K^{n}$-isomorphism $\psi_{m_{P_{1}}}(x)$ a dilatation, if it satisfies $\psi_{m_{P_{i}}}(x)=\left(z_{1}, . ., z_{n}\right), z_{i}=y_{i}+\pi^{m_{i}} x_{i}$, for each $i=1,2, . ., n$. We define the dilatation of $f(x)$ at $P_{1}$ induced by $\psi_{m_{P_{1}}}(x)$, as

$$
f_{P_{1}}(x):=\pi^{-e_{P_{1}}} f\left(\psi_{m_{P_{1}}}(x)\right)
$$

where $e_{P_{1}}$ is the minimum order of $\pi$ in the coefficients of $f\left(\psi_{m_{P_{1}}}(x)\right)$. We call the $K$-hypersurface $V_{f_{P_{1}}}$ the dilatation of $V_{f}$ at $P_{1}$ induced by $\psi_{m_{P_{1}}}(x)$, the number $e_{P_{1}}$ the arithmetic multiplicity of $f(x)$ at $P_{1}$ by $\psi_{m_{P_{1}}}(x)$ and the set $S\left(f_{P_{1}}\right)$, the lifting of $\operatorname{Sing}_{\overline{f_{P_{1}}}}\left(\mathbb{F}_{q}\right)$, the first generation of descendants of $P_{1}$.

Given a sequence of dilatations $\left(\psi_{m_{P_{k}}}(x)\right)_{k}$, we inductively define $e_{P_{1}, \ldots, P_{k}}$, $f_{P_{1}, \ldots, P_{k}(x)}$, and $S\left(f_{P_{1}, \ldots, P_{k}}\right)$ as follows:

$$
f_{P_{1}, \ldots, P_{k}}(x)=\left\{\begin{array}{l}
f(x) \quad \text { if } k=0, \\
\pi^{-e_{P_{1}}, \ldots, P_{k}} f_{P_{1}, \ldots, P_{k-1}}\left(\psi_{m_{P_{k}}}(x)\right) \quad \text { if } k \geqq 1,
\end{array}\right.
$$

where $P_{k} \in S\left(f_{P_{1}, \ldots, P_{k-1}}\right)$, and $e_{P_{1}, \ldots, P_{k}}$ is the minimum order of $\pi$ in the coefficients of $f_{P_{1}, \ldots, P_{k-1}}\left(\psi_{m_{P_{k}}}(x)\right)$. The union of the sets $S\left(f_{P_{1}, \ldots, P_{k}}\right)$ is called the $k$-generation of descendants of $P_{1}$.

In this paper we shall use several types of dilatations, i.e., dilatations with different $m$ 's, but the specific value of $m$ will be clear from the context. The dilatations were introduced by A. Néron (cf. [13, sect. 18). These transformations play an important role in the process of desingularization of the closed fiber of a scheme over a discrete valuation ring whose generic fiber is non-singular. A modern exposition of the Néron $\pi$-desingularization can be found in [1], sect. 4 .

Now, we review Igusa's stationary phase formula, from the point of view of the dilatations. For that, we fix the $m_{P_{k}}$ 's equal to $(1, . ., 1) \in \mathbb{N}^{n}$ in $(2.2)$.

Let $\bar{D}$ be a subset of $\mathbb{F}_{q}^{n}$ and $D$ its preimage under the canonical homomorphism $\mathcal{O}_{K} \longrightarrow \mathcal{O}_{K} / \pi \mathcal{O}_{K} \cong \mathbb{F}_{q}$. Let $S(f, D)$ denote the subset of $R^{n}$ (the set of representatives of $\mathbb{F}_{q}^{n}$ in $\left.\mathcal{O}_{K}^{n}\right)$ mapped bijectively to the set $\operatorname{Sing}_{\bar{f}}\left(\mathbb{F}_{q}\right) \cap \bar{D}$. We use the simplified notation $S(f)$, in the case of $D=\mathcal{O}_{K}^{n}$. Also we define

$$
\begin{gathered}
\nu(\bar{f}, D):=q^{-n} \operatorname{Card}\left\{\bar{P} \in \bar{D} \mid \bar{P} \notin V_{\bar{f}}\left(\mathbb{F}_{q}\right)\right\} \\
\sigma(\bar{f}, D):=q^{-n} \operatorname{Card}\left\{\bar{P} \in \bar{D} \mid \bar{P} \text { is a non-singular point of } V_{\bar{f}}\left(\mathbb{F}_{q}\right)\right\} .
\end{gathered}
$$

In order to simplify the notation, we shall use $\nu(\bar{f})$ and $\sigma(\bar{f})$ instead of $\nu(\bar{f}, D)$ and $\sigma(\bar{f}, D)$, respectively, if $D=\mathcal{O}_{K}^{n}$.

With all this, we are able to establish Igusa's stationary phase formula for $\pi$-adic integrals: 
Igusa's Stationary Phase Formula ([10, p. 177]).

$$
\begin{aligned}
\int_{D}|f(x)|_{K}^{s}|d x|= & \nu(\bar{f}, D)+\sigma(\bar{f}, D) \frac{\left(1-q^{-1}\right) q^{-s}}{\left(1-q^{-1} q^{-s}\right)} \\
& +\sum_{P \in S(f, D)} q^{-n-e_{P} s} \int_{\mathcal{O}_{K}^{n}}\left|f_{P}(x)\right|_{K}^{s}|d x|,
\end{aligned}
$$

where $\operatorname{Re}(s)>0$. Formula (2.3) is obtained in the following form. Suppose that $\bar{D}=\left\{\overline{P_{1}}, . ., \overline{P_{N}}\right\}$ and let $P_{i}$ be the lifting of $\overline{P_{i}}$. Then the set $D$ is the disjoint union $\bigcup_{P} D_{P}$, where $\bar{P}=\left(\overline{y_{1}}, . ., \overline{y_{n}}\right) \in \bar{D}$ and $D_{P}$ is defined as

$$
D_{P}=\left\{\left(x_{1}, \ldots, x_{n}\right) \in D \mid x_{i}=y_{i}+\pi z_{i}, z_{i} \in \mathcal{O}_{K}, \quad i=1,2, . ., n\right\} .
$$

Thus

$$
\int_{D}|f(x)|_{K}^{s}|d x|=\sum_{\bar{P}} \int_{D_{P}}|f(x)|_{K}^{s}|d x|=\sum_{\bar{P}} q^{-n-e_{P} s} \int_{\mathcal{O}_{K}^{n}}\left|f_{P}(x)\right|_{K}^{s}|d x| .
$$

The integrals corresponding to the $P$ 's for which $\bar{P} \notin V_{\bar{f}}\left(\mathbb{F}_{q}\right)$ are easily computable. The integrals corresponding to the $P$ 's for which $\bar{P}$ is a non-singular point of $V_{\bar{f}}\left(\mathbb{F}_{q}\right)$ are computed using the implicit function theorem (cf. [10], p. 177).

We define $I_{k}$ inductively as follows:

$$
I_{1}:=\left\{P_{1} \mid P_{1} \in S(f, D)\right\},
$$

and

$$
I_{k}:=\left\{\left(P_{1}, . ., P_{k}\right) \mid\left(P_{1}, . ., P_{k-1}\right) \in I_{k-1} \text { and } P_{k} \in S\left(f_{P_{1}, . ., P_{k-1}}\right)\right\}, \quad k \geq 2 .
$$

With the above notation and by iterating the stationary phase formula, we obtain the following expansion for $Z(f, s)$ (cf. [10], p. 178):

$$
\begin{aligned}
Z(f, s)= & \nu(\bar{f}, D)+\sigma(\bar{f}, D) \frac{\left(1-q^{-1}\right) q^{-s}}{\left(1-q^{-1} q^{-s}\right)} \\
& +\sum_{k \geq 1} q^{-k n}\left(\sum_{\left(P_{1}, \ldots P_{k}\right) \in I_{k}} \nu\left(\bar{f}_{P_{1}, \ldots P_{k}}\right) q^{-E\left(P_{1}, \ldots P_{k}\right) s}\right) \\
& +\frac{\left(1-q^{-1}\right) q^{-s}}{\left(1-q^{-1} q^{-s}\right)} \sum_{k \geq 1} q^{-k n}\left(\sum_{\left(P_{1}, \ldots P_{k}\right) \in I_{k}} \sigma\left(\bar{f}_{P_{1}, \ldots P_{k}}\right) q^{-E\left(P_{1}, \ldots P_{k}\right) s}\right),
\end{aligned}
$$

where $E\left(P_{1}, . ., P_{k}\right):=e_{P_{1}}+\ldots+e_{P_{1}, \ldots P_{k}}$. Expansion (2.4) converges absolutely on the semiplane $\operatorname{Re}(s)>0$.

Now, we summarize some ideas on Néron $\pi$-desingularization (see [13], sect. $17,18)$ to be used in the next sections. Let $f(x) \in \mathcal{O}_{K}[x]$ be a polynomial, and $P \in V_{f}\left(\mathcal{O}_{K}\right)$. Néron introduced the following measure of singularity at $P$ :

$$
l(f, P):=\operatorname{Inf}_{i=1, \ldots, n}\left(v\left(\frac{\partial f}{\partial x_{i}}(P)\right)\right) .
$$

The Jacobian criterion implies that $P$ is a smooth point of $V_{f}(K)$ if and only if $l(f, P)$ is finite. $\bar{P}$ is a smooth point of $V_{\bar{f}}\left(\mathbb{F}_{q}\right)$ if and only if $l(f, P)=0$. In this paper, we introduce the following measure of singularity at an integer point $P$, satisfying $P \notin \operatorname{Sing}_{f}\left(\mathcal{O}_{K}\right)$. 
Definition 2.1. Let $f(x) \in \mathcal{O}_{K}[x]$ be a polynomial and $P \in \mathcal{O}_{K}^{n}$ a point, such that $P \notin \operatorname{Sing}_{f}\left(\mathcal{O}_{K}\right)$. We define

$$
L(f, P):=\operatorname{Inf}\left(v(f(P)), v\left(\frac{\partial f}{\partial x_{1}}(P)\right), \ldots, v\left(\frac{\partial f}{\partial x_{n}}(P)\right)\right) .
$$

Let us observe that $L(f, P)=0$ if and only if

$$
\overline{f(x)}=\alpha_{0}+\sum_{j=1}^{n} \alpha_{j}\left(x_{j}-\overline{a_{j}}\right)+(\text { degree } \geq 2),
$$

where $P=\left(a_{1}, . ., a_{n}\right), \alpha_{j} \in \mathbb{F}_{q}^{*}$ for some $j=0,1,2, . ., n$. We also observe that $l(f, p)=L(f, p)$ if $P \in V_{f}\left(\mathcal{O}_{K}\right)$. The integer $L(f, P)$ has properties similar to those of $l(f, P)$. This integer appears naturally associated to Igusa's stationary phase, as we shall see later on.

We denote by $A_{r}, r=\left(r_{1}, . ., r_{n}\right) \in(\mathbb{N} \backslash\{0\})^{n}$, the set

$$
A_{r}:=\left\{x \in \mathcal{O}_{K}^{n} \mid v\left(x_{i}\right) \geq r_{i}, i=1, . ., n\right\} .
$$

From a geometrical point of view, $A_{r}$ is a polydisc in $\mathcal{O}_{K}^{n}$ centered at the origin. The complement of $A_{r}$ in $\mathcal{O}_{K}^{n}$ is denoted by $A_{r}^{c}$.

The following proposition is a simple reformulation of proposition 17 in sect. 17 of [13]. However, for our convenience, we prove it below.

Proposition 2.2 (Néron, 13, sect. 17, prop. 17). Let $f(x) \in \mathcal{O}_{K}[x]$ be a polynomial, $P \in \mathcal{O}_{K}^{n}$ an absolutely algebraically isolated singularity of the hypersurface $V_{f}$, and let $D \subseteq \mathcal{O}_{K}^{n}$ be a subset such that $D \cap\left(P+A_{r}\right)=\emptyset$, for some $r \in(\mathbb{N} \backslash\{0\})^{n}$. Then

$$
L(f, Q) \leq C(f, D), \text { for every } Q \in D,
$$

where the constant $C(f, D)$ depends only on $f$ and $D$.

Proof. Without loss of generality, we may suppose that the point $P$ is the origin of $K^{n}$. The hypothesis that the origin of $K^{n}$ is an absolutely algebraically isolated singularity and the Hilbert Nullstellensatz imply that

$$
\pi^{m_{i}} x_{i}^{t_{i}}=A_{i, 0}(x) f(x)+\sum_{j=1}^{n} A_{i, j}(x) \frac{\partial f}{\partial x_{j}}(x),
$$

for some $m_{i}, t_{i} \in \mathbb{N}$, with $t_{i} \neq 0$, and some polynomials $A(x)_{i, j} \in \mathcal{O}_{K}[x]$, for each $i=1,2, . ., n$. Now, let $Q=\left(q_{1}, . ., q_{n}\right)$ be a point of $D$. Since $D \cap A_{r}=\emptyset$, there exists a coordinate $j_{0}$ such that $v\left(q_{j_{0}}\right)<r_{j_{0}}$. From (2.5), with $x=Q$ and $i=j_{0}$, we obtain

$$
m_{j_{0}}+t_{j_{0}} r_{j_{0}} \geq m_{j_{0}}+t_{j_{0}} v\left(q_{j_{0}}\right) \geq L(f, Q) .
$$

Thus, it is sufficient to take $C(f, D) \geq \operatorname{Max}_{i}\left\{m_{i}+t_{i} r_{i}\right\}$.

The following result is a generalization of proposition 18 (cf. [13, sect. 18) of A. Néron.

Proposition 2.3 (Néron, 13, sect. 18, prop. 18). Let $f(x) \in \mathcal{O}_{K}[x]$ be a polynomial and $P=\left(b_{1}, . ., b_{n}\right) \in \mathcal{O}_{K}^{n}$ a point such that $P \notin \operatorname{Sing}_{f}\left(\mathcal{O}_{K}\right)$. Then there exists a minimal non-negative integer $\mu(f, P)$ such that the polynomial

$$
f_{P}(x)=\pi^{-e_{\mu, P}} f\left(P+\pi^{\mu} x\right),
$$


where $e_{\mu, P}$ is the minimum order of $\pi$ in the coefficients of $f\left(P+\pi^{\mu} x\right)$, satisfies

$$
\overline{f_{P}(x)}=\alpha_{0}+\sum_{i=1}^{n} \alpha_{i} x_{i}, \quad \alpha_{i} \in \mathbb{F}_{q}^{*}, \text { for some } i=0,1,2, . ., n .
$$

Furthermore, $\mu(f, P) \leq L(f, P)+1$.

Proof. By induction on $L(f, P)$.

Case $L(f, P)=0$. In this case, we take $\mu(f, P)=1 \leq L(f, P)+1$.

Case $L(f, P)>0$. In this case, we have

$$
f(x)=\alpha_{0}+\sum_{i=1}^{n} \alpha_{i}\left(x_{i}-b_{i}\right)+(\text { degree } \geq 2),
$$

where $\alpha_{i} \equiv 0 \bmod \pi, i=0,1, . ., n$. Thus

$$
f(P+\pi x)=\pi\left(\alpha_{0}^{\prime}+\sum_{i=1}^{n} \alpha_{i} x_{i}+\pi(\text { degree } \geq 2)\right) .
$$

We consider two cases according to whether $\alpha_{0}^{\prime} \not \equiv 0 \bmod \pi$ or not.

Case $1\left(\alpha_{0}^{\prime} \not \equiv 0 \bmod \pi\right)$. In this case, we have

$$
f(P+\pi x)=\pi f_{P}(x)
$$

where

$$
f_{P}(x)=\alpha_{0}^{\prime}+\sum_{i=1}^{n} \alpha_{i} x_{i}+\pi(\text { degree } \geq 2) .
$$

Therefore, $\overline{f_{P}(x)}=\overline{\alpha_{0}^{\prime}} \in \mathbb{F}_{q}^{*}$, and $\mu(f, P)=1 \leq L(f, P)$.

Case $2\left(\alpha_{0}^{\prime} \equiv 0 \bmod \pi\right)$. In this case, we have

$$
f(P+\pi x)=\pi^{2}\left(\alpha_{0}^{\prime \prime}+\sum_{i=1}^{n} \alpha_{i}^{\prime} x_{i}+(\text { degree } \geq 2)\right)=\pi^{e_{\mu, P}} f_{P}(x),
$$

where $e_{\mu, P} \geq 2$. Thus

$$
f(P)=\pi^{e_{\mu, P}} f_{P}(0)
$$

and

$$
\frac{\partial f}{\partial x_{i}}(P)=\pi^{e_{\mu, P}-1} \frac{\partial f_{P}}{\partial x_{i}}(0), \quad i=1, . ., n .
$$

Hence $L\left(f_{P}, 0\right) \leq L(f, P)-1$. The result follows from the induction hypothesis by observing that $\mu(f, P)=\mu\left(f_{P}, 0\right)+1 \leq L\left(f_{P}, 0\right)+1+1 \leq L(f, P)+1$.

As a consequence of the two above results, we obtain the following lemma.

Lemma 2.4. Let $f(x) \in \mathcal{O}_{K}[x]$ be a polynomial such that the origin of $K^{n}$ is an absolutely algebraically isolated singularity. Let $A_{r}$ be a polydisc with $r \in(\mathbb{N} \backslash\{0\})^{n}$. Then there exists $\gamma=\gamma(f, r) \in \mathbb{N} \backslash\{0\}$, such that the polynomial

$$
f_{Q}(x)=\pi^{-e_{Q, \gamma}} f\left(Q+\pi^{\gamma} x\right),
$$

satisfies the condition that $\overline{f_{Q}(x)}$ is a non-zero constant or a linear polynomial with or without constant term, for all $Q \in A_{r}^{c}$. 


\section{Rationality of Igusa's LOCAL ZETA FUnCTIONS OF SEMIQUASIHOMOGENEOUS POLYNOMIALS}

In this section, we prove the rationality of the Igusa local zeta function of semiquasihomogeneous polynomials.

Lemma 3.1. Let $D \subseteq \mathcal{O}_{K}^{n}$ be the preimage under the canonical homomorphism $\mathcal{O}_{K} \longrightarrow \mathcal{O}_{K} / \pi \mathcal{O}_{K}$ of a subset $\bar{D} \subseteq \mathbb{F}_{q}^{n}$. Let $f(x) \in \mathcal{O}_{K}[x]$ be a polynomial such that the origin of $K^{n}$ is an absolutely algebraically isolated singularity of $V_{f}(K)$. If $D \cap A_{r}=\emptyset$, for some $r \in(\mathbb{N} \backslash\{0\})^{n}$, then the integral $Z(f, D, s)=\int_{D}|f|_{K}^{s}|d x|$ is a rational function of $q^{-s}$. More precisely,

$$
Z(f, D, s)=\frac{L\left(q^{-s}, D\right)}{1-q^{-1} q^{-s}} .
$$

Furthermore, the polynomial $L\left(q^{-s}, D\right)$ can be effectively computed.

Proof. By applying the stationary phase formula $m+1=\gamma(f, r)$ times, we obtain

$$
\begin{gathered}
Z(f, D, s)=\nu(\bar{f}, D)+\sigma(\bar{f}, D) \frac{\left(1-q^{-1}\right) q^{-s}}{\left(1-q^{-1} q^{-s}\right)} \\
+\sum_{k=1}^{m} q^{-k n}\left(\sum_{\left(P_{1}, P_{2}, \ldots P_{k}\right) \in I_{k}} \nu\left(\bar{f}_{P_{1}, \ldots P_{k}}\right) q^{-E\left(P_{1}, \ldots P_{k}\right) s}\right) \\
+\frac{\left(1-q^{-1}\right) q^{-s}}{\left(1-q^{-1} q^{-s}\right)} \sum_{k=1}^{m} q^{-k n}\left(\sum_{\left(P_{1}, P_{2}, \ldots P_{k}\right) \in I_{k}} \sigma\left(\bar{f}_{P_{1}, \ldots P_{k}}\right) q^{-E\left(P_{1}, \ldots P_{k}\right) s}\right) \\
+\sum_{\left(P_{1}, . ., P_{m+1}\right) \in I_{m+1}} q^{-(m+1) n-E\left(P_{1}, \ldots P_{m+1}\right) s} \int_{\mathcal{O}_{K}^{n}}\left|f_{P_{1}, . ., P_{m+1}}(x)\right|_{K}^{s}|d x| .
\end{gathered}
$$

On the other hand, we have

$$
f\left(P_{1}+P_{2} \pi+\ldots+P_{m} \pi^{m+1}+\pi^{m+2} x\right)=\pi^{E\left(P_{1}, \ldots, P_{m}\right)} f_{P_{1}, \ldots, P_{m}}(x),
$$

and $P_{1}+P_{2} \pi+\ldots .+P_{m} \pi^{m+1} \in A_{r}^{c}$. Because $m+2 \geq \gamma(f, r)$, it follows from lemma 2.4 and (3.3) that the $\mathbb{F}_{q}$-hypersurface defined by $\overline{f_{P_{1}, \ldots ., P_{m}}(x)}$ is smooth or empty. Then $I_{m+1}=\emptyset$, and the integral $Z(f, D, s)$ is a rational function of form (3.1).

Lemma 3.2. Let $f(x) \in \mathcal{O}_{K}[x]$ be a polynomial such that the origin of $K^{n}$ is an absolutely algebraically isolated singularity of $V_{f}(K)$. Then the integral $Z\left(f, A_{r}^{c}, s\right)=$ $\int_{A_{r}^{c}}|f|^{s}|d x|$ is a rational function of $q^{-s}$. More precisely,

$$
Z\left(f, A_{r}^{c}, s\right)=\frac{L\left(q^{-s}\right)}{1-q^{-1} q^{-s}} .
$$

Furthermore, the polynomial $L\left(q^{-s}\right)$ can be computed effectively.

Proof. We introduce a family $\mathcal{L}$ of sets defined as follows. For each subset $B$ of $\{1, \ldots, n\}$ and each $a=\left(a_{1}, \ldots, a_{n}\right) \in \mathbb{N}^{n}$ satisfying $0 \leq a_{i}<r_{i}$ if $i \in B$, we define

$$
D(B, a):=\left\{\begin{array}{l}
\left\{x \in A_{r}^{c} \mid v\left(x_{i}\right)=a_{i} \text { if } i \in B\right\}, \quad \text { if } B \neq \emptyset, \\
\emptyset, \quad \text { if } B=\emptyset .
\end{array}\right.
$$


The family $\mathcal{L}$ is closed under intersections, and its union is $A_{r}^{c}$. We denote by $J$ the set of indices $\{(B, a)\}$ and by $\mathcal{P}(J)$ the family of subsets of $J$ with $i$ elements.

Hence

$$
Z\left(f, A_{r}^{c}, s\right)=\sum_{i=1}^{\operatorname{Card}\{J\}}(-1)^{i-1} \sum_{T \in \mathcal{P}(J)_{i}} \int_{D(T)}|f|^{s}|d x|,
$$

where $D(T):=\bigcap_{(B, a) \in T} D(B, a)$. From (3.6) and the fact that the family $\mathcal{L}$ is closed under intersections, it follows that in order to prove the theorem, it is sufficient to prove that any integral of type

$$
\int_{D(B, a)}|f|^{s}|d x|, \quad B \neq \emptyset
$$

is a rational function of the form $L\left(q^{-s}, D(B, a)\right) /\left(1-q^{-1} q^{-s}\right)$, where the numerator polynomial is effectively computable. For that, we make the following change of variables in (3.7):

$$
x=\psi_{(B, a)}(y), \text { where } x_{i}= \begin{cases}\pi^{a_{i}} y_{i} \text { if } i \in B, \\ y_{i} \text { if } i \notin B .\end{cases}
$$

We obtain

$$
\int_{D(B, a)}|f|^{s}|d x|=q^{-e_{(B, a)} s-d_{(B, a)}} \int_{D^{\prime}(B, a)}\left|f_{B}\right|^{s}|d y|,
$$

where $d_{(B, a)}=\sum_{i \in B} a_{i}, f_{B}(y)$ is the dilatation of $f$ induced by (3.8) and

$$
D^{\prime}(B, a)=\prod_{i=1}^{n} R_{i},
$$

where $R_{i}=\mathcal{O}_{K}$ if $i \notin B$ and $R_{i}=\mathcal{O}_{K}^{*}$ if $i \in B$.

On the other hand, $\phi(y)$ defines a $K$-isomorphism of $K^{n} \longrightarrow K^{n}$; thus the $K$-singular locus of $V_{f}$ is mapped bijectively on the $K$-singular locus of $V_{f_{B}}$. Therefore, the polynomial $f_{B}$ and the set $D^{\prime}(B, a), B \neq \emptyset$, satisfy the conditions of lemma 3.1. Thus the integral $\int_{D^{\prime}(B, a)}\left|f_{B}\right|^{s}|d x|$ is a rational function of $q^{-s}$, and its numerator can be computed effectively.

Proposition 3.3. Let $F(x)=f(x)+\pi^{m} g(x) \in \mathcal{O}_{K}[x]$ be a polynomial such that the origin of $K^{n}$ is an absolutely algebraically isolated singularity of $V_{F}(K)$. Let $D \subseteq \mathcal{O}_{K}^{n}$ be the preimage under the canonical homomorphism $\mathcal{O}_{K} \longrightarrow \mathcal{O}_{K} / \pi \mathcal{O}_{K}$ of a subset $\bar{D} \subseteq \mathbb{F}_{q}^{n}$, and $A_{r}$ a polydisc such that $D \cap A_{r}=\emptyset, r \in(\mathbb{N} \backslash\{0\})^{n}$. There exists $\alpha(f, D)$, effectively computable, such that if $m \geq \alpha(f, D)$ then

$$
Z(F, D, s)=Z(f, D, s) .
$$

Proof. By virtue of lemma 2.4, there exists a $\gamma_{0}=\gamma(F, r)$ such that the reduction modulo $\pi$ of the polynomial

$$
F_{P_{1}, \ldots, P_{n}}(x)=\pi^{-E_{F}\left(P_{1}, . ., P_{n}\right)} F\left(P_{1}+P_{2} \pi+\ldots .+P_{n} \pi^{n}+\pi^{n+1} x\right)
$$

is a non-zero constant or a linear polynomial for every $n \geq \gamma_{0}$ and any $P_{1}+P_{2} \pi+$ $\ldots .+P_{n} \pi^{n} \in A_{r}^{c}$. This implies implies that $I_{\gamma_{0}+1}=\emptyset$. So the integral $Z(F, D, s)$ admits a finite expansion of type (3.2).

We set

$$
g_{P_{1}, \ldots, P_{n}}(x):=\pi^{-E_{g}\left(P_{1}, . ., P_{n}\right)} g\left(P_{1}+P_{2} \pi+\ldots .+P_{n} \pi^{n}+\pi^{n+1} x\right),
$$


where $E_{g}\left(P_{1}, . ., P_{n}\right)$ is the minimum order of $\pi$ in the coefficients of

$$
g\left(P_{1}+P_{2} \pi+\ldots+P_{n} \pi^{n}+\pi^{n+1} x\right) .
$$

With this notation, we have that

$$
\begin{gathered}
F\left(P_{1}+P_{2} \pi+\ldots .+P_{n} \pi^{n}+\pi^{n+1} x\right)=\pi^{E_{f}\left(P_{1}, . ., P_{n}\right)} f_{P_{1}, \ldots, P_{n}}(x) \\
+\pi^{E_{g}\left(P_{1}, . ., P_{n}\right)+m} g_{P_{1}, \ldots, P_{n}}(x) .
\end{gathered}
$$

Now, we choose

$$
\alpha(f, D):=\operatorname{Max}_{\left(P_{1}, . ., P_{\gamma_{0}}\right) \in I_{\gamma_{0}}}\left\{1+E_{f}\left(P_{1}, . ., P_{\gamma_{0}}\right)\right\} .
$$

Thus, if $m \geq \alpha(f, D)$, then

$$
\begin{gathered}
\overline{F_{P_{1}, P_{2}, . ., P_{k}}(x)}=\overline{f_{P_{1}, P_{2}, \ldots, P_{k}}(x)}, \\
E_{F}\left(P_{1}, P_{2}, . ., P_{k}\right)=E_{f}\left(P_{1}, P_{2}, . ., P_{k}\right), \quad 1 \leq k \leq \gamma_{0} .
\end{gathered}
$$

From (3.13) it follows that

1) $\nu(\bar{f}, D)=\nu(\bar{F}, D)$ and $I_{1}(f)=I_{1}(F)$, because $\bar{f}(x)=\bar{F}(x)$.

2) For every $1 \leq k \leq \gamma_{0}$

$$
\nu\left(\overline{f_{P_{1}, . ., P_{k}}}\right)=\nu\left(\overline{F_{P_{1}, . ., P_{k}}}\right) \text {, for every }\left(P_{1}, . ., P_{k}\right) \in I_{k}(f)=I_{k}(F) .
$$

3) For every $1 \leq k \leq \gamma_{0}$

$$
I_{k+1}(f)=I_{k+1}(F)
$$

because $I_{k}(f)=I_{k}(F)$ and $\overline{f_{P_{1}, . ., P_{k}}(x)}=\overline{F_{P_{1}, . ., P_{k}}}(x)$ for every $\left(P_{1}, . ., P_{k}\right) \in I_{k}(f)=$ $I_{k}(F)$.

Therefore $I_{\gamma_{0}+1}(f)=I_{\gamma_{0}+1}(F)=\emptyset$, and $Z(f, s, D)$ admits a finite expansion of type (3.2). Now it is easy to see that $Z(F, D, s)=Z(f, D, s)$.

Lemma 3.4. Let $F(x)=f(x)+\pi^{m} g(x) \in \mathcal{O}_{K}[x]$ be a polynomial such that the origin of $K^{n}$ is an absolutely algebraically isolated singularity of $V_{F}(K)$. Let $A_{r}$ be a polydisc, with $r \in(\mathbb{N} \backslash\{0\})^{n}$. There exists $\alpha(f, r)$, effectively computable, such that if $m \geq \alpha(f, r)$ then

$$
Z\left(F, A_{r}^{c}, s\right)=Z\left(f, A_{r}^{c}, s\right) .
$$

Proof. By (3.6), it is sufficient to prove the lemma for integrals of the type (3.7). We choose $\alpha(f, r)$ satisfying

$$
\alpha(f, r) \geqq \operatorname{Max}_{(B, a)}\left\{e_{f,(B, a)}\right\} .
$$

With the above condition, it is sufficient to prove that

$$
\int_{D^{\prime}(B, a)}\left|F_{B}\right|^{s}|d x|=\int_{D^{\prime}(B, a)}\left|f_{B}\right|^{s}|d x|,
$$

with $D^{\prime}(B, a)$ as in $(3.9)$ and $F_{B}(x)=f_{B}(x)+\pi^{m-e_{f,(B, a)}} g\left(\psi_{(B, a)}(x)\right)$.

The result follows from (3.14) and proposition 3.3. Finally, we observe that $\alpha(f, r)$ is given by

$$
\alpha(f, r)=\operatorname{Max}_{(B, a)}\left\{e_{f,(B, a)}+\alpha\left(f_{B}, D^{\prime}(B, a)\right)\right\} .
$$


Theorem 3.5. Let $F(x) \in K[x]$ be a semiquasihomogeneous polynomial whose quasihomogeneous part $f(x)$ has weight $d$ and exponents $\alpha_{1}, . ., \alpha_{n}$. Then Igusa's local zeta function of $F(x)$ is a rational function of $q^{-s}$. More precisely,

$$
Z(F, s)=\frac{L\left(q^{-s}\right)}{\left(1-q^{-1} q^{-s}\right)\left(1-q^{-|\alpha|} q^{-d s}\right)}
$$

where $\alpha=\left(\alpha_{1}, \ldots, \alpha_{n}\right)$. Furthermore, the polynomial $L\left(q^{-s}\right)$ can be computed effectively.

Proof. We may suppose without loss of generality that $F(x) \in \mathcal{O}_{K}[x]$. By decomposing $\mathcal{O}_{K}^{n}$ as the disjoint union of $A_{\alpha}$ and $A_{\alpha}^{c}$ and using the fact that $F(x)$ is a semiquasihomogeneous polynomial, we obtain

$$
\begin{aligned}
Z(F, s) & =\int_{A_{\alpha}}|F|_{K}^{s}|d x|+\int_{A_{\alpha}^{c}}|F|_{K}^{s}|d x| \\
& =q^{-|\alpha|-d s} \int_{\mathcal{O}_{K}^{n}}\left|F_{1}\right|_{K}^{s}|d x|+\int_{A_{\alpha}^{c}}|F|_{K}^{s}|d x|,
\end{aligned}
$$

where $F_{1}(x)=f(x)+\pi^{m_{1}} H_{1}(x)$, with $m_{1} \geq 1$. Now, iterating formula (3.16) $m+1$ times, we obtain

$$
Z(F, s)=Z\left(F, A_{\alpha}^{c}, s\right)+\sum_{k=1}^{m} q^{k(-|\alpha|-d s)} Z\left(F_{k}, A_{\alpha}^{c}, s\right)+q^{(m+1)(-|\alpha|-d s)} Z\left(F_{m+1}, s\right),
$$

where $F_{k}(x)=f(x)+\pi^{m_{k}} H_{k}(x)$, with $m_{k} \longrightarrow \infty$. By lemma 3.4, there exists $\gamma_{0}=\gamma_{0}(f, \alpha)$, effectively computable, such that

$$
Z\left(F_{k}, A_{\alpha}^{c}, s\right)=Z\left(f, A_{\alpha}^{c}, s\right), \text { for every } m_{k} \geq \gamma_{0} .
$$

Thus from (3.17), we have

$$
\begin{gathered}
Z(F, s)=Z\left(F, A_{\alpha}^{c}, s\right)+\sum_{k=1}^{\gamma_{0}-1} q^{k(-|\alpha|-d s)} Z\left(F_{k}, A_{\alpha}^{c}, s\right) \\
+Z\left(f, A_{\alpha}^{c}, s\right) q^{\left(\gamma_{0}+1\right)(-|\alpha|-d s)} \frac{1}{1-q^{-|\alpha|-d s}} .
\end{gathered}
$$

By lemma 3.2 the integrals $Z\left(f, A_{\alpha}^{c}, s\right)$ and $Z\left(F_{k}, A_{\alpha}^{c}, s\right)$ are rational functions of $q^{-s}$ of the form $\frac{L\left(q^{-s}\right)}{1-q^{-1} q^{-s}}$, where the polynomial numerator can be effectively computed.

We observe that if $f$ is a quasihomogeneous polynomial, its local zeta function is given by $Z(f, s)=\frac{Z\left(f, A_{\alpha}^{c}, s\right)}{1-q^{-}|\alpha|-d s}$. The integral $Z\left(f, A_{\alpha}^{c}, s\right)$ can be computed using lemma 3.2 .

As a consequence of theorem 3.5, we obtain the following three corollaries. In the first corollary we shall use freely the notions of Newton polyhedron, non-degeneracy with respect to a Newton polyhedron, and Bernstein polynomial; the corresponding definitions can be found in references [1], 2].

Corollary 3.6. Let $K$ be a global field and let $F(x) \in K[x]$ be a semiquasihomogeneous polynomial whose quasihomogeneous part $f(x)$ has weight $d$ and exponents $\alpha_{1}, . ., \alpha_{n}$. Then for every non-archimedean valuation $v$ of $K$, Igusa's local zeta function of $F(x)$ on the completion $K_{v}$ of $K$ is a rational function of the form 
(3.15). If $K$ is a number field and $F(x)$ is non-degenerate with respect to its Newton polyhedron, then the real parts of the poles of the zeta function $Z(F, s)$ are roots of the Bernstein polynomial of $F(x)$.

Proof. Since $F(x)$ has an absolutely algebraically isolated singularity at the origin of $K^{n}$, the Hilbert Nullstellensatz implies that for all non-archimedean valuations $v$ of $K$, the origin of $K_{v}^{n}$ is an absolutely algebraically isolated singularity of $V_{f}\left(K_{v}\right)$. Thus by the proof of theorem 3.5, the denominator of the the local zeta function $Z(f, s)$ on $K_{v}$ is equal to $\left(1-q^{-1} q^{-s}\right)\left(1-q^{-|\alpha|} q^{-d s}\right)$. Thus the real parts of the poles of $Z(F, s)$ are among the values $-1,-|\alpha| / d$. If $K$ is a number field, and $F(x)$ is non-degenerate with respect to its Newton polyhedron, theorem C.2.2.3 of [2] implies that -1 and $-|\alpha| / d$ are roots of the Bernstein polynomial of $F(x)$.

The following corollary gives a bound for the number of solutions of a conguence attached to a semiquasihomogeneous polynomial with coefficients in a ring of integers of a global field. This corollary follows directly from the relation existing between the Igusa local zeta function and the Poincaré series $P(t)$ (see the introduction) and corollary 3.6.

Corollary 3.7. Let $K$ be a global field and $\mathcal{O}_{K}$ its ring of integers. Let $F(x) \in$ $\mathcal{O}_{K}[x]$ be a semiquasihomogeneous polynomial whose quasihomogeneous part $f(x)$ has weight $d$ and exponents $\alpha_{1}, \ldots, \alpha_{n}$. Then for every non-archimedean valuation $v$ of $K$, the number of solutions $N_{j}(F, v)$ of the congruence

$$
F(x) \equiv 0 \bmod \pi^{j} \mathcal{O}_{K_{v}},
$$

where $\mathcal{O}_{K_{v}}$ is the ring of integers of the completion $K_{v}$, satisfies

$$
\lim \sup _{j \rightarrow \infty} N_{j}(F, v)^{1 / j} \leq \begin{cases}q^{n-|\alpha| / d} & \text { if }|\alpha| / d \leq 1, \\ q^{n-1} & \text { if }|\alpha| / d>1 .\end{cases}
$$

The following corollary follows directly from theorem 3.5. We use the notation established in the introduction.

Corollary 3.8. Let $f(x, y) \in K[x, y]$ be an absolutely analytically irreducible polynomial, such that the origin of $K^{2}$ is an absolutely algebraically isolated singularity of the plane curve $V_{f_{d}}$. Then the Igusa local zeta function $Z(f, s)$ is a rational function of $q^{-s}$ of form (3.15).

Example 3.9. In this example we compute the local zeta function for a polynomial of type $f(x, y)=\alpha x^{n}+\beta y^{m}, \alpha, \beta \in \mathcal{O}_{K}$, where $n, m>1$ are relatively prime. Suppose that the characteristic of $K$ does not divide both $n$ and $m$. Furthermore, without loss of generality, we may suppose that $\alpha \in \mathcal{O}_{K}^{*}$. In the case of charactersitic zero, the Poincaré series $P(t)$ associated to this type of polynomials were explicitly computed by Goldman (cf. [6], thm. 1).

Using the observation made after the proof of theorem 3.5, we have

$$
Z(f, s)=\frac{1}{1-q^{-(n+m)-m n s}} \int_{A^{c}}|f|_{K}^{s}|d x d y|,
$$

where $A=\left\{(x, y) \in \mathcal{O}_{K}^{2} \mid v(x) \geq m, \quad v(y) \geq n\right\}$. The complement $A^{c}$ of $A$ is the disjoint union of the following three sets:

$$
D_{1}=\left\{(x, y) \in \mathcal{O}_{K}^{2} \quad \mid v(x)<m, \quad v(y) \geq n\right\},
$$




$$
\begin{aligned}
& D_{2}=\left\{(x, y) \in \mathcal{O}_{K}^{2} \quad \mid v(x)<m, \quad v(y)<n\right\} . \\
& D_{3}=\left\{(x, y) \in \mathcal{O}_{K}^{2} \quad \mid v(x) \geq m, \quad v(y)<n\right\} .
\end{aligned}
$$

Thus from (3.19), we obtain

$$
Z(f, s)=\frac{1}{1-q^{-(n+m)-m n s}}\left\{Z\left(f, D_{1}, s\right)+Z\left(f, D_{2}, s\right)+Z\left(f, D_{3}, s\right)\right\} .
$$

Next, we compute the integrals $Z\left(f, D_{1}, s\right), Z\left(f, D_{2}, s\right), Z\left(f, D_{3}, s\right)$.

Computation of $Z\left(f, D_{1}, s\right)$. First, we observe that

$$
|f(x, y)|=\left|\alpha x^{n}+\beta y^{m}\right|=\left|x^{n}\right|, \quad x, y \in D_{1} .
$$

Therefore

$$
Z\left(f, D_{1}, s\right)=\int_{D_{1}}|f|_{K}^{s}|d x d y|=\sum_{k=0}^{m-1} \int_{\left\{(x, y) \in D_{1} \mid v(x)=k, v(y) \geq n\right\}}|x|_{K}^{n s}|d x d y| .
$$

Thus

$$
Z\left(f, D_{1}, s\right)=\left(1-q^{-1}\right) q^{-n} \sum_{k=0}^{m-1} q^{-k n s-k}
$$

Computation of $Z\left(f, D_{2}, s\right)$. We set $L(i, j):=j m-i n+v(\beta)$. The set $D_{2}$ can be decomposed as the union of three disjoint subsets $D_{2,1}, D_{2,2}, D_{2,3}$, as follows:

$$
\begin{aligned}
& D_{2,1}:=\left\{(x, y) \in D_{2} \mid L(v(x), v(y))>0\right\}, \\
& D_{2,2}:=\left\{(x, y) \in D_{2} \mid L(v(x), v(y))<0\right\}, \\
& D_{2,3}:=\left\{(x, y) \in D_{2} \mid L(v(x), v(y))=0\right\} .
\end{aligned}
$$

Thus $Z\left(f, D_{2}, s\right)=Z\left(f, D_{2,1}, s\right)+Z\left(f, D_{2,2}, s\right)+Z\left(f, D_{2,3}, s\right)$, where

$$
Z\left(f, D_{2,1}, s\right)=\left(1-q^{-1}\right)^{2} \sum_{i, j} q^{-i-j-n i s},
$$

where $i, j$ satisfy $L(i, j)>0$ and $0 \leqq i<m, 0 \leqq j<n$,

$$
Z\left(f, D_{2,2}, s\right)=\left(1-q^{-1}\right)^{2} \sum_{i, j} q^{-i-j-(v(\beta)+m j) s},
$$

where $i, j$ satisfy $L(i, j)<0$ and $0 \leqq i<m, 0 \leqq j<n$, and

$$
Z\left(f, D_{2,3}, s\right)=\sum_{i, j} q^{-i-j-n i s} \int_{\mathcal{O}_{K}^{\times 2}}\left|\alpha x^{n}+\mu y^{m}\right|_{K}^{s}|d x d y|,
$$

where $\beta=\pi^{v(\beta)} \mu, \mu \in \mathcal{O}_{K}^{\times}, i, j$ satisfy $L(i, j)=0$ and $0 \leqq i<m, 0 \leqq j<n$. Using the stationary phase formula, we compute the integral in the right side of (3.24); thus

$$
Z\left(f, D_{2,3}, s\right)=\left(\nu\left(\bar{f}, \mathcal{O}_{K}^{\times 2}\right)+\frac{\sigma\left(\bar{f}, \mathcal{O}_{K}^{\times 2}\right)\left(1-q^{-1}\right) q^{-s}}{1-q^{-1-s}}\right) \sum_{i, j} q^{-i-j-n i s} .
$$

We denote by $[x]$ the integer part of a real number $x$. We set $v(\beta)=g n+r$, $0 \leq r<n$. 
Computation of $Z\left(f, D_{3}, s\right)$. We set

$$
\begin{aligned}
& D_{3,1}:=\left\{(x, y) \in \mathcal{O}_{K}^{2} \quad \mid \quad v(x) \geq m+\left[\frac{v(\beta)}{n}\right]+1, \quad v(y)<n\right\}, \\
& D_{3,2}:=\left\{(x, y) \in \mathcal{O}_{K}^{2} \quad \mid m \leq v(x) \leq m+\left[\frac{v(\beta)}{n}\right], \quad v(y)<n\right\} .
\end{aligned}
$$

Then $D_{3}=D_{3,1} \cup D_{3,2}$ (disjoint union), and $Z\left(f, D_{3}, s\right)=Z\left(f, D_{3,1}, s\right)+$ $Z\left(f, D_{3,2}, s\right)$. To compute $Z\left(f, D_{3,1}, s\right)$, we observe that

$$
|f(x, y)|=\left|\alpha x^{n}+\beta y^{m}\right|=\left|\beta y^{m}\right|, \quad x, y \in D_{3,1} .
$$

Thus

$$
\begin{aligned}
Z\left(f, D_{3,1}, s\right) & =\int_{D_{3,1}}|f(x, y)|_{K}^{s}|d x d y| \\
& =\left(1-q^{-1}\right) q^{-\left(m+\left[\frac{v(\beta)}{n}\right]+1\right)} \sum_{k=0}^{n-1} q^{-(v(\beta)+m k) s-k}
\end{aligned}
$$

The set $D_{3,2}$ can be decomposed as the union of three disjoint subsets $D_{3,2,1}$, $D_{3,2,2}, D_{3,2,3}$, as follows:

$$
\begin{aligned}
& D_{3,2,1}:=\left\{(x, y) \in D_{3,2} \mid L(v(x), v(y))>0\right\}, \\
& D_{3,2,2}:=\left\{(x, y) \in D_{3,2} \mid L(v(x), v(y))<0\right\}, \\
& D_{3,2,3}:=\left\{(x, y) \in D_{3,2} \mid L(v(x), v(y))=0\right\} .
\end{aligned}
$$

Thus $Z\left(f, D_{3,2}, s\right)=Z\left(f, D_{3,2,1}, s\right)+Z\left(f, D_{3,2,2}, s\right)+Z\left(f, D_{3,2,3}, s\right)$, and

$$
Z\left(f, D_{3,2,1}, s\right)=\left(1-q^{-1}\right)^{2} \sum_{i, j} q^{-i-j-n i s},
$$

where $i, j$ satisfy $L(i, j)>0$ and $m \leqq i<m+\left[\frac{v(\beta)}{n}\right], 0 \leqq j<n$,

$$
Z\left(f, D_{3,2,2}, s\right)=\left(1-q^{-1}\right)^{2} \sum_{i, j} q^{-i-j-(v(\beta)+m j) s},
$$

where $i, j$ satisfy $L(i, j)<0$ and $m \leqq i<m+\left[\frac{v(\beta)}{n}\right], 0 \leqq j<n$, and

$$
Z\left(f, D_{3,2,3}, s\right)=\left(\nu\left(\bar{f}, \mathcal{O}_{K}^{\times 2}\right)+\frac{\sigma\left(\bar{f}, \mathcal{O}_{K}^{\times 2}\right)\left(1-q^{-1}\right) q^{-s}}{1-q^{-1-s}}\right) \sum_{i, j} q^{-i-j-n i s},
$$

where $i, j$ satisfy $L(i, j)=0$ and $m \leqq i<m+\left[\frac{v(\beta)}{n}\right], 0 \leqq j<n$.

Example 3.10. A polynomial of the form $f(x)=\sum_{i} \beta_{i} x_{i}^{n_{i}}, \beta_{i} \in \mathcal{O}_{K}$, is called a diagonal polynomial. We set $d:=$ l.c.m. $\left\{n_{i}\right\}$, and $\alpha_{i}:=\frac{d}{n_{i}}, i=1, . ., n$. If the characteristic of $K$ does not divide any $n_{i}$, then the diagonal polynomials are quasihomogeneous polynomials with exponents $\alpha_{i}:=\frac{d}{n_{i}}, i=1, . ., n$, and weight $d$. Thus the local zeta function of a diagonal polynomial is a rational function of the form (3.13). Wang and others have studied the Poincaré series $P(t)$ associated to this class of polynomials (cf. 14], thm. 1). 


\section{ACKNOWLEDGEMENTS}

The author wishes thank to the following institutions for their support: Universidad Autónoma de Bucaramanga, Academia Colombiana de Ciencias Exactas, Físicas y Naturales, and COLCIENCIAS. The author also thanks IMPA for their support and hospitality during the summer of 1997, when part of this work was done. The author also wishes to thank the referee for his or her useful comments, which led to an improvement of this work.

\section{REFERENCES}

[1] Arnold V., Varchenko A. and Gussein-Zadé S., Singularités des applications differéntiables, vol. 2, Editions Mir, Moscow, 1986.

[2] Briancon J., Granger M., Maisonobe Ph., Miniconi M., Algorithme de calcul du polynôme de Bernstein: cas non-dégénéré, Ann. Inst. Fourier 39 (1989), 553-610. MR 91k:32040

[3] Denef J., The rationality of the Poincaré series associated to the p-adic points on a variety, Invent. Math. 77 (1984), 1-23. MR 86c:11043

[4] Denef J., Report on Igusa's local zeta functions, Seminaire Bourbaki 741 (1990-1991), Astérisque No. 201-302 (1991), 359-386. MR 93g:11119

[5] Denef J., Poles of p-adic complex powers and Newton Polyhedra, Nieuw Archief voor Wiskunde 13 (1995), 289-295. MR 96m:11106

[6] Goldman J., Number of solutions of congruences: Poincaré series for algebraic curves, Adv. in Math. 62 (1986), 68-83. MR 88b:11035

[7] Igusa J.-I., Complex powers and asymptotic expansions I, J. Reine Angew. Math. 268/269 (1974), 110-130. MR 50:241

[8] Igusa J.-I., Complex powers and asymptotic expansions II, J. Reine Angew. Math. 278/279 (1975), 307-321. MR 53:8018

[9] Igusa J.-I., Complex powers of irreducible algebroid curves, Geometry Today, Roma 1989, Progress in Math. 60, Birkhäuser, 1985, pp. 201-230. MR 88j:11084

[10] Igusa J.-I., A stationary phase formula for p-adic integrals and its applications (Conf. in honor of S. S. Abhyankar), Algebraic geometry and its applications, Springer-Verlag, 1994, pp. 175-194. MR 95a:11104

[11] Loeser F., Fonctions d'Igusa p-adiques, polynômes de Bernstein, et polyèdres de Newton, J. Reine Angew. Math. 412 (1990), 75-96. MR 92c:11139

[12] Meuser D., On the poles of a local zeta function for curves, Invent. Math. 73 (1983), 445-465. MR 85i:14014

[13] Néron A., Modèles minimaux des variétés abéliennes sur corps locaux et globaux, Pub. Math. I.H.E.S. 21 (1964). MR 31:3423

[14] Wang J., On Poincaré series for diagonal forms, Proc. Amer. Math. Soc. 116 (1992), 607611. MR 93a:11032

Universidad Autónoma de Bucaramanga, Laboratorio de Computo Especializado, A.A. 1642 , Bucaramanga, Colombia

Current address: 6351 SW 43rd Street, Miami, Florida 33155

E-mail address: wzuniga@bumanga.unab.edu.co 